\title{
Percutaneous Nephrolithotomy in a Patient with Mainz Pouch II Urinary Diversion: A Case Report
}

\author{
Stavros Sfoungaristos ${ }^{1}$, loannis Mykoniatis ${ }^{1}$, Evangelos Poulios ${ }^{1}$, \\ Dimitrios Paikos², Dimitrios Hatzichristou' \\ $11^{\text {st }}$ Department of Urology, Aristotle University, Thessaloniki, Greece; \\ ${ }^{2}$ Deparment of Gastroenterology, G. Gennimatas Hospital, Thessaloniki, Greece
}

Received June 18, 2016; Accepted November 14, 2016.

Key words: Mainz pouch - Percutaneous nephrolithotomy - Urolithiasis Lithotripsy

\begin{abstract}
Mainz pouch II is a reliable and viable technique of continent urinary diversion. Patients are at increased risk of long-term complications including urolithiasis of the upper urinary tract and reservoir. We report the case of a 67-year-old male with prior Mainz pouch II due to invasive bladder cancer treated for a large renal calculus. Percutaneous nephrolithotomy (PCNL) was successfully performed. Stone management in these type of patients is of increased interest due to existed "anatomical challenges" concerning the access and safety during the procedure. To our knowledge this is the first case of PCNL in a patient with Mainz pouch II that has been reported in the literature.
\end{abstract}

Mailing Address: Stavros Sfoungaristos, MD., PhD., $1^{\text {st }}$ Department of Urology, Aristotle University, Thessaloniki, Greece; Phone: +302 310963 396; e-mail: sfoungaristosst@gmail.com 


\section{Introduction}

Mainz pouch II or sigma-rectum pouch represents a continent urinary diversion for patients undergoing a radical cystectomy (Fisch et al., 1993). Sigmoid colon is utilized to create a low pressure and high capacity reservoir. Patients with urinary diversions are at increased risk for upper tract stones formation as well as calculi within the diversion segment (Okhunov et al., 2011). There are several treatment options for managing renal calculi. Following the establishment of minimal invasion techniques, percutaneous nephrolithotomy (PCNL) is considered the preferred option for treating renal stones in patients with urinary diversion.

Although PCNL is an efficient and safe technique, it may be a demanding procedure in case of urinary diversion. Gaining percutaneous access is a challenging step since identification of the neo-ureteral orifice and retrograde opacification of the collecting system is commonly difficult. There are several reports in the literature presenting the outcomes of PCNL in a variety of diversion types (ElNahas et al., 2006; Hertzig et al., 2013). However, data regarding the outcomes of PCNL in patients with Mainz pouch II is missing. In the present report, we describe the management of a large renal stone in a patient with Mainz pouch II urinary diversion by PCNL.

\section{Case report}

A 67-year-old Caucasian male was referred to our Department of Urology for definitive treatment of a large renal calculus. According to his past medical history, he underwent radical cystectomy and Mainz pouch II urinary diversion due to invasive bladder cancer 8 years ago. He also underwent an open ureterolithotomy for stone removal 2 years ago. The patient was admitted to another institution due to acute pyelonephritis 2 months ago. Obstruction of the left kidney was identified by ultrasound and a nephrostomy tube was inserted. A computed

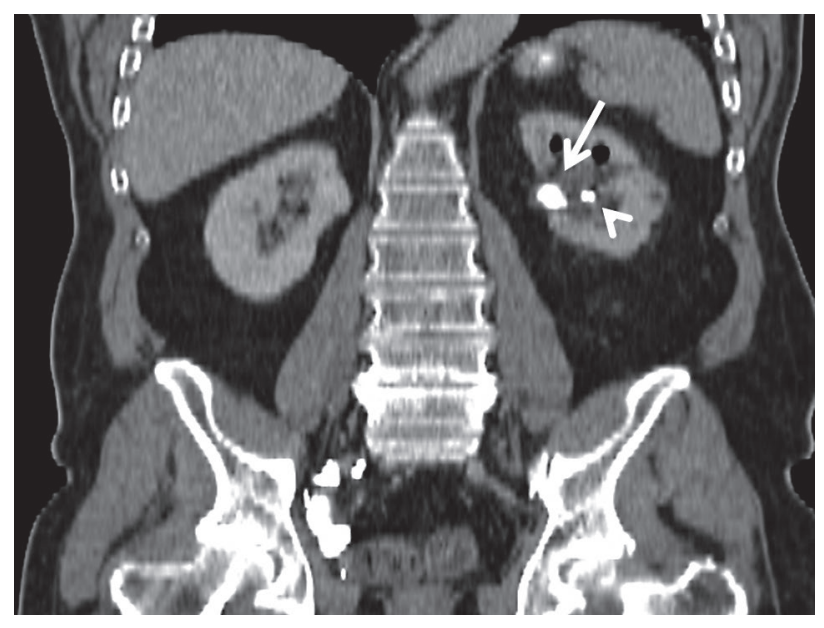

Figure 1 - Computed tomography scan revealing the stone (arrow - renal stone; arrowhead nephrostomy tube). 
tomography showed a $21 \times 13 \times 15 \mathrm{~mm}$ stone within the left renal pelvis (Figure 1 ). Surgical options were discussed and PCNL was considered the optimal option. Preoperative urine culture revealed multi-drug resistant Pseudomonas aeruginosa and Meropenem was initiated $24 \mathrm{~h}$ prior the procedure.

After inducing general anesthesia, the patient was placed in prone position. Nephrostomy tube was utilized and antegrade pyelography was performed for

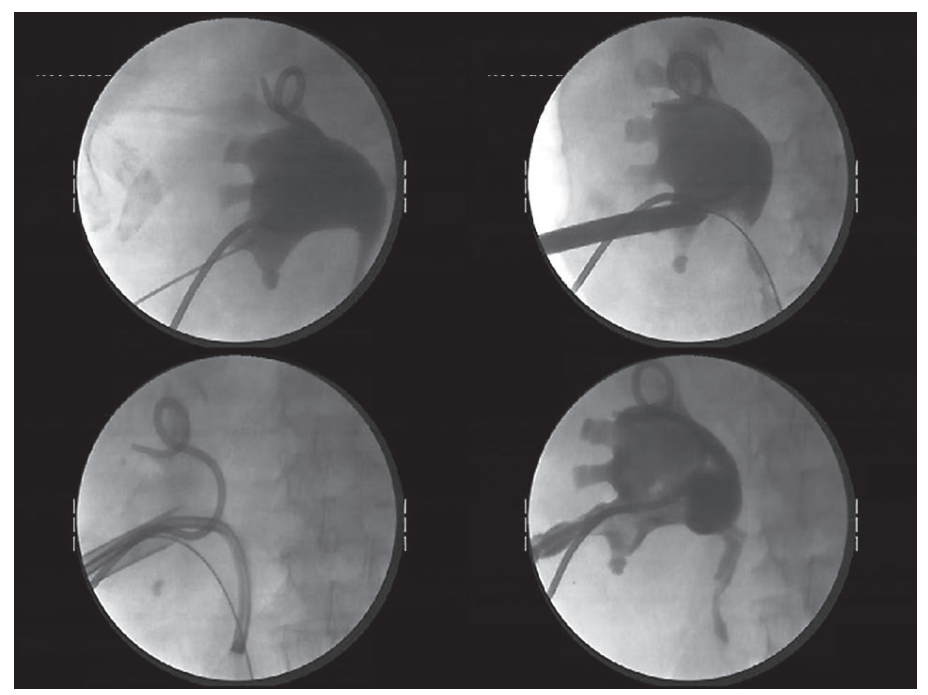

Figure 2 - Figure 2a (upper left) - an $18 G$ needle inserted in lower calyx; Figure $2 b$ (upper right) - obtaining the tract with 30F balloon dilation; Figure $2 c$ (lower left) - inspection of the ureter with flexible nephroscope; Figure $2 d$ (lower right) - insertion of Council catheter and antegrade pyelography.

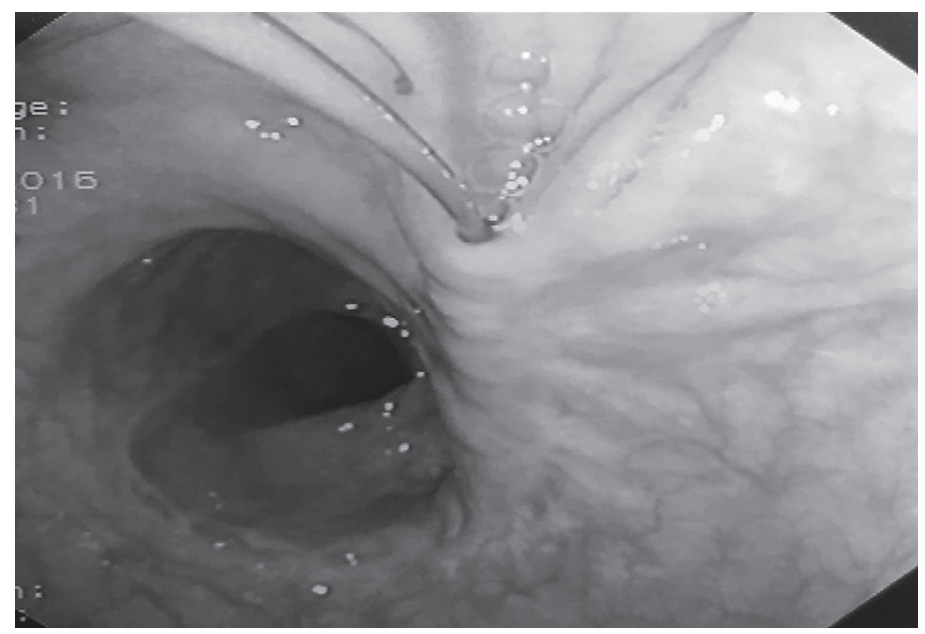

Figure 3 - Neo-ureteral orifice and safety wire. 
the identification of optimal calyx. Puncture of posterior lower calyx was chosen using the "bull's eye" technique. After confirming the site of the needle within the collecting system (Figure 2a), a 0.035 -inch hydrophilic-coated angled-tip guidewire was inserted. A $5 \mathrm{~F}$ "hockey stick" catheter was then advanced guiding the wire down the ureter into the Mainz pouch. At this point, the guidewire was grasped and pulled out through the rectum by flexible colonoscope (Figure 3), obtaining a "through and through" wire. Guidewire was replaced with Amplatz 0.038-inch extra-stiff wire. A gas tube was placed into the pouch through the rectum. The

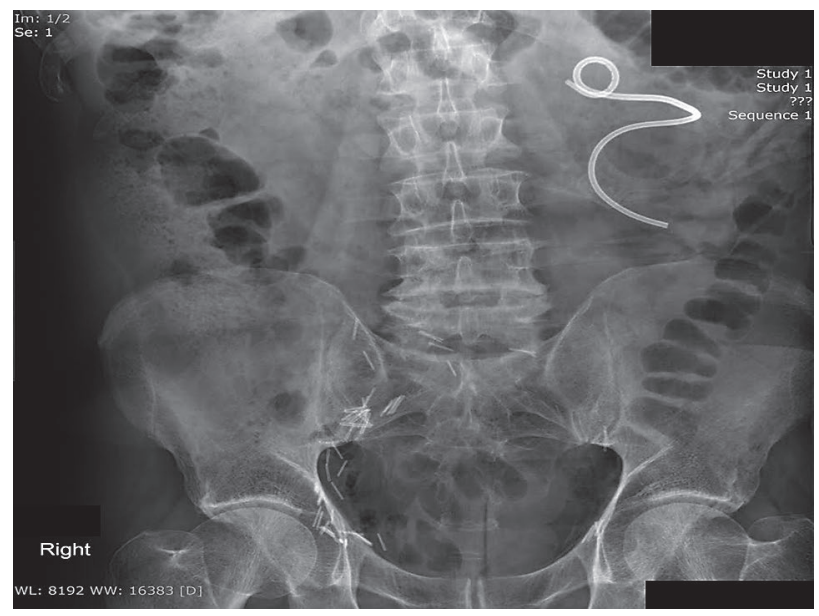

Figure 4 - Postoperative KUB (kidney-ureter-bladder).

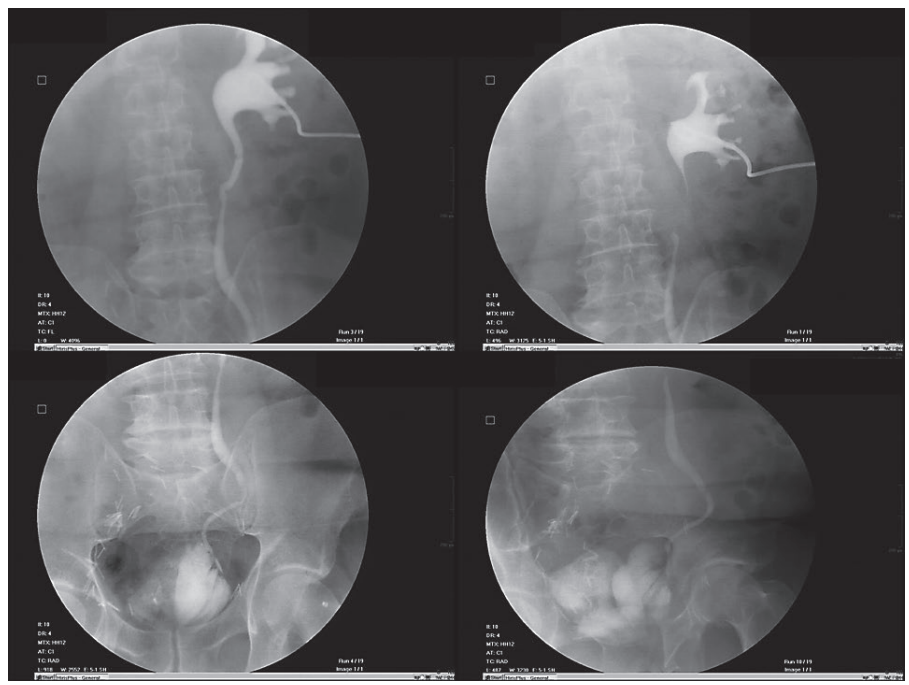

Figure 5 - Postoperative nephrostomography. 
percutaneous tract was established by $30 \mathrm{~F}$ balloon dilation (Figure $2 \mathrm{~b}$ ). The calculus was identified by rigid nephroscope, and fragmentation performed by ultrasonic lithotripter. Significant fragments were retrieved by duckbill stone grasper. Following stone removal, inspection of the upper calyces and ureter was performed by flexible nephroscope (Figure 2c). Spotted remaining fragments were removed by tipless nitinol basket while a significant fragment, identified within proximal ureter, was fragmented by Ho:YAG laser. Intra-operative stone-free status was confirmed by fluoroscopy and antegrade pyelography. An $18 \mathrm{~F}$ council catheter was placed into the renal pelvis (Figure 2d). Sequential exchange and withdrawal of wires and catheters were performed antegradely to avoid retrograde microbial migration from the pouch to the collecting system.

Postoperative course was uneventful. Gas tube was removed the $1^{\text {st }}$ postoperative day. Patient was discharged the $2^{\text {nd }}$ post-operative day after removal of council catheter. Nephrostomy tube remained closed but not removed. Stone-free status was confirmed by KUB (kidney-ureter-bladder) the $2^{\text {nd }}$ post-operative day (Figure 4). One month after the procedure, a nephrostomography was performed to confirm stone-free status and ureteral patency (Figure 5). Nephrostomy tube was safely removed. Stone analysis revealed a mixed type stone, composed by struvite and apatite.

\section{Discussion}

Mainz pouch II technique was first described in 1993 (Fisch et al., 1993). The rectosigmoid reservoir is a low pressure pouch providing continence, protection of the upper urinary tract and minimal morbidity (Bastian et al., 2004). Compared to other types of continent diversions, Mainz II results to notable decrease in mean and peak bowel contraction pressures, as confirmed in postoperative rectodynamic studies (Okhunov et al., 2011).

It is well-established that patients undergoing urinary diversion are at amplified risk of calculi formation. Reported prevalence varies between 3\% and 43\%. Risk factors include postoperative anatomical changes resulting to urinary stasis and mucus reflux into the upper tract, bacterial colonization and diversion-associated metabolic disorders (Okhunov et al., 2011).

As it has been previously reported, PCNL represents the preferred treatment option for large renal stones in patients with urinary diversion (Okhunov et al., 2011). Identification of the neo-ureteral orifices and retrograde pyelography are challenging steps in these cases. Ultrasound-guided or blind puncture is usually needed in order to obtain primary access and opacify the collecting system. In our case, ultrasound-guided nephrostomy insertion had preceded the operation. The nephrostomy tube was utilized for antegrade pyelography and lower calyx puncture under fluoroscopy guidance.

Under the contribution and assistance of gastroenterologists, we obtain a "through and through" wire. This step represents a significant part of the 
procedure since it offers maximal safety by providing a retrograde pathway.

Stone analysis revealed a mixed type stone, consisting by struvite and calcium phosphate. As it has been reported before, the above stone types represent the most common types of diversion stones (Okhunov et al., 2011).

Surgical management of renal stone disease in patients with urinary diversion requires detailed evaluation and individualized consideration depending on stone location and burden, diversion type and surgeon's experience. To the best of our knowledge, this is the first case of percutaneous nephrolithotomy in a patient with Mainz pouch II urinary diversion that has been reported in the English literature. Although the experience regarding renal stone management in patients with Mainz pouch II is minimal, we may report that PCNL can be performed with safety and maximal efficacy. The combination of rigid and flexible scopes is mostly needed to obtain postoperative stone-free status. A "through and through" wire is strongly advised since it may provide retrograde and antegrade access to the collecting system.

\section{References}

Bastian, P. J., Albers, P., Haferkamp, A., Schumacher, S., Müller, S. C. (2004) Modified ureterosigmoidostomy (Mainz pouch II) in different age groups and with different techniques of ureteric implantation. BJU Int. 94, 345-349.

El-Nahas, A. R., Eraky, I., el-Assmy, A. M., Shoma, A. M., el-Kenawy, M. R., Abdel-Latif, M., Mosbah, A., Abol-Enein, H., Shaaban, A. A., Mohsen, T., el-Kappany, H. A. (2006) Percutaneous treatment of large upper tract stones after urinary diversion. Urology 68, 500-504.

Fisch, M., Wammack, R., Müller, S. C., Hohenfellner, R. (1993) The Mainz pouch II (sigma rectum pouch). J. Urol. 149, 258-263.

Hertzig, L. L., Iwaszko, M. R., Rangel, L. J., Patterson, D. E., Gettman, M. T., Krambeck, A. E. (2013) Urolithiasis after ileal conduit urinary diversion: a comparison of minimally invasive therapies. J. Urol. 189, 2152 2157.

Okhunov, Z., Duty, B., Smith, A. D., Okeke, Z. (2011) Management of urolithiasis in patients after urinary diversions. BJU Int. 108, 330-336. 